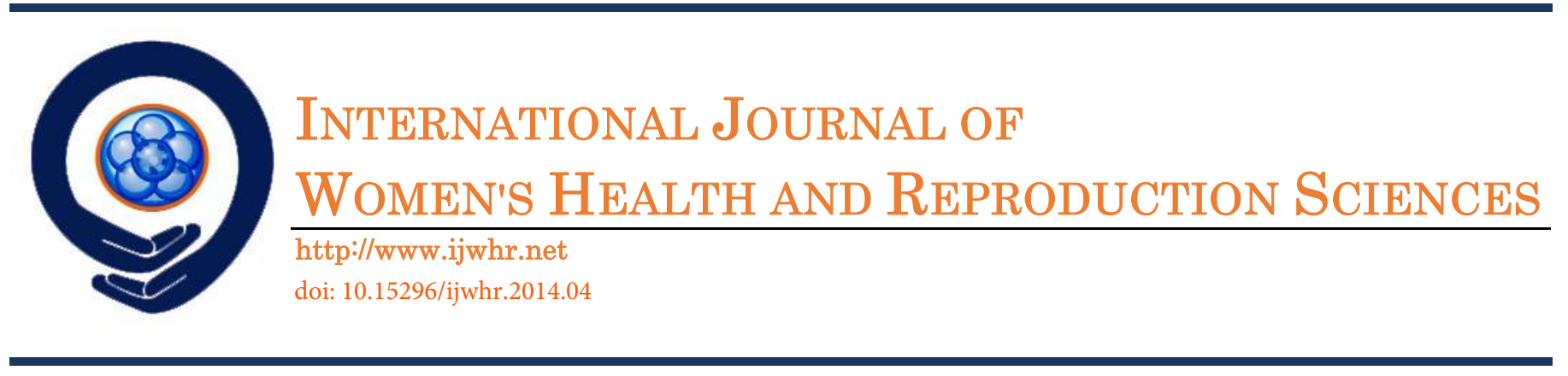

\title{
Assessing the Relative Contribution of Causes Leading to Cesarean Section Among Women Who had Referred to ShahidMadani Clinic in Marand City
}

\author{
Zahra Ghanbari ${ }^{1}$, Rana Shokouhmand ${ }^{2}$, HakimehKhatoon Asadi ${ }^{2}$, \\ Shirin Naghizadeh ${ }^{2}$, Fatemeh Chupani ${ }^{3}$
}

Article History:

Received September 2013

Accepted October 2013

Available online January 2014

Keywords:

Cesarean Section

Fear of Labor Pain

Indication of Cesarean Section

Corresponding Author:

Zahra Ghanbari, Faculty of

Medical Sciences, Islamic Azad

University Tabriz Branch, Tabriz, Iran

Tel: +989143057376

Email: dr_zahra2009@yahoo.com

\begin{abstract}
Objectives: Cesarean section is a surgical procedure and it is performed when the normal vaginal delivery is not available or vaginal delivery is dangerous for mother or fetus. In other words, there are some special indications for cesarean section. In the past decade the rate of cesarean delivery in the absence of clear indications has increased. This subject suggests an intensive research and attention. This study has compared the causes of cesarean delivery among women.

Material and methods: 100 women who had referred to Shahid Madani Clinic in Marand city, were asked based on questionnaire that included information about patients and a list of causes that could lead women to a cesarean delivery.Thereafter, the contribution of each cause was assessed.

Results: Fear of labor pain is the most common cause of cesarean delivery (35\%) while having history of previous caesarean section, fear of disturbing the anatomy of external genitalia , medical indication for caesarean section and cultural beliefescaused with lower rate in comparsion to fear of labor pain. were placed in lower classes.

Conclusion: This study showed that, several factors may lead women to caesarean delivery; The fear of labor pain is the most common factor that could be reduced by proper training and holding childbirth preparation classes for pregnant women.
\end{abstract}

1-Faculty of Medical Sciences, Islamic Azad University Tabriz Branch, Tabriz, Iran.

2-Marand Health Network System, Tabriz University of Medical Sciences, Tabriz, Iran.

3-Faculty of Medicine, Department of Midwifery, Tabriz University of Medical Sciences, Tabriz, Iran. 


\section{Introduction:}

Cesarean section is the delivery of a baby through a surgical incision made in the mother's abdomen and uterus. In past centuries,cesarean section was done to save fetus in women who were full-term but had died before birth.

The first modern cesarean was conducted by Dr. James Barry in South Africa on July 1826(1).

Cesarean section is performed when the normal vaginal childbirth is not proper for the mother or for some reasons childbirth must be done sooner; in other words, there is an indication for cesarean delivery. Some of the indications for cesarean ,including breech or transverse position of the fetus, placenta previa, fetal respiratory problems, HIV positive mothers, baby with a malformation such as NTD(Neural Tube Defect), obstruction of the vaginal canal with a large fibroid, history of previous cesarean section. Despite the advances in medical science, cesarean section is accompanied by complication during labor and after that such as atonic uterus and bleeding, uterine rupture, increasing the risk of bowel adhesions and damage to the urinary tract (2). In the past three decades caesarean section has increased in the world so this issue requires careful consideration. According to the Disease control and prevention center of America in 2009, 33\% of women had a cesarean delivery. The cesarean section rate has been increased nearly 60 percent since 1996 in United States(2).

Recent studies have also shown that babies who are born by cesarean section are not exposed to the bacteria in the birth canal, this can lead to a loss of colonization of bacteria that can be useful in the future for the baby's health(3).

According to official statistics in Iran, cesarean delivery rate is about 25 to $35 \%$, while according to unofficial statistic, cesarean section rate in Iran is five times more in compartion to other parts of the world . Reasons of increased rate of caesarean sections are as follows:

1: medical advances and technology: by reducing the risk of surgery and cesarean section for fetalas well as increase of neonatal care.

2: This option is selected by women on the basis of cultural beliefs such as the lack of pressure on the baby's head.

3: Some experts say that major cause is women fear of labor pain.

4: Scientific and medical causes: baby's heart failure and the fetal placental detachment, etc.

\section{Material \& Methods:}

In a cross-sectional study about 100 women who referred to Shahid Madani Clinic in Marand city from march 2013 to September 2013 and had a cesarean section were selected .They were asked based on a questionnaire containing personal information and causes which could lead to cesarean delivery such as fear of labor pain, history of previous cesarean, fear of disturbing anatomy of genital tract, cultural beliefs and indications for cesarean section.Besides,the contribution of each cause was assessed.

\section{Results:}

The mean age of women was 27.33 years .Comparisons have shown that fear of labor pain was 35\%, history of previous cesarean section was $22 \%$, fear of disturbing the anatomy of genital was $15 \%$,cultural beliefs $13 \%$, and indications for cesarean section was $15 \%$.(Figure 1 )

\section{Discussion:}

In study of Saisto in 2002 interested on complications of cesarean section including hemorrhage and longer hospital stays in mothers and increased risk of neonatal respiratory morbidity (4).

According to Manacker and et al study in $2007,32 \%$ of all births in United States occurred by cesarean section(5).

Nieminen and et al studies showed that relationship between fear of labor and preferred of cesarean section (6).Ryding and et al in 1991 showed that fear of childbirth is common reasons for demanding cesarean section in patient(7).

In study of Saisto and et al in 2001 on psychosocial characteristics of women fearing vaginal childbirth showed that the personalities of womens contributed to the women's fear of vaginal delivery(8). 
In a case-control study that was performed by Ryding and et al in 1991 among 1981 swedish-speaking women,97 patient were deliverd by cesarean section and fear of childbirth of these cause were compred with the same features in 194 controls,matched for age and parity.women deliverd by CS reported a fear of chidbirth (9).

In study of Rouhe and et al in 2009 fear of childbirth is one of the most common cause of cesarean section in women(10).

Hildingsson and et al in 2002 determind that fear of childbirth was the only women's wish for cesarean section(11).

In our study among 100 women that had a cesarean section ,fear of childbirth was the most common cause of cesarean delivery.

\section{Conclusion :}

Cesarean rates have increased in the past years that these rises have different reasons such as having a history of previous cesarean section, medical indications for cesarean, cultural beliefs, the fear of labor pain and disturbing the genital anatomy. In this study the fear of the pain of natural childbirth was greatest reason for cesarean delivery,that can be reduced by proper training and holding childbirth preparation classes for pregnant women.

\section{Conflict of interest statement:}

We declare that we have no conflict of interest.

\section{Acknowledgments:}

We have no acknowledgements to disclose.

Figure1: Assessement of the contribution of causes leading to cesarean section.

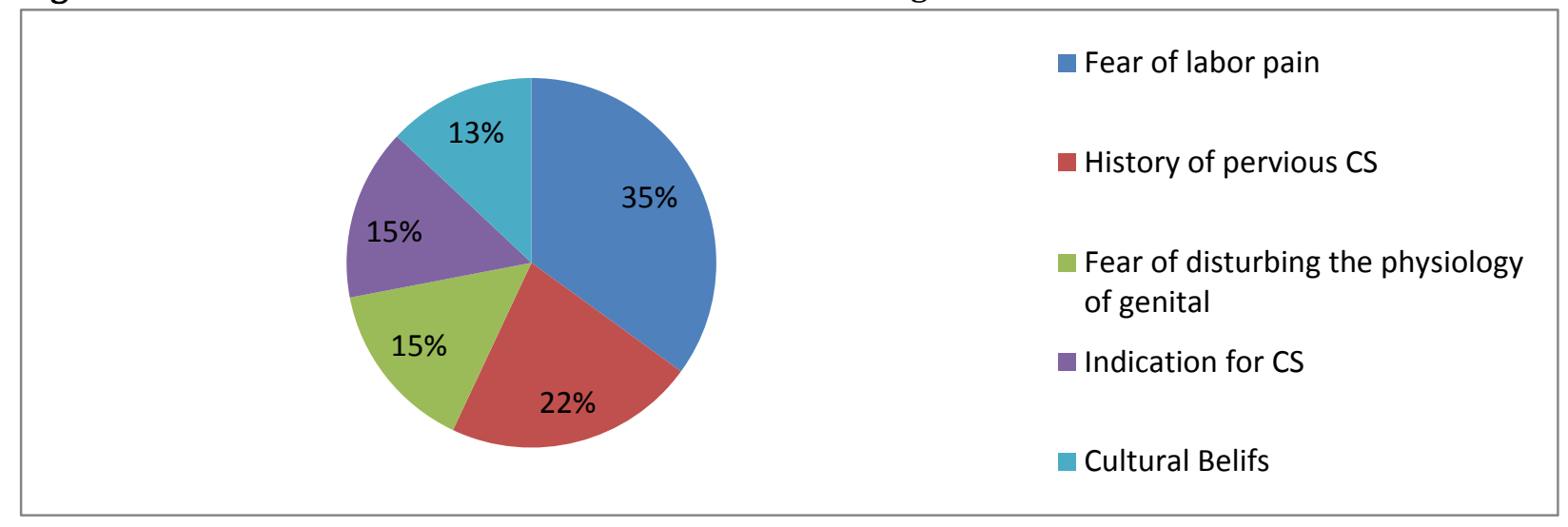




\section{References:}

1. Cornje HS. Clinical obstetrics. A South African perspective. 2012; 3.345.

2. Mukhergee SN. The Journal of Obstetrics and Gynecology of India. 2006; 56(4): 298-300.

3. Trask L.The risk of cesarean section for the baby . 2013.

4. Saisto T.Obstetric,Psychosocial,And Pain Related Back ground,and Treatment of fear of childbirth. 2002.

5. Menacker F,Hamilton BE.Recent trends in cesarean delivery in the United States.NCHS Data Brief. 2010; 35.1-8.

6. Nieminen K,Stephansson O,Ryding EL.Womens fear of childbirth and preference for cesarean section - a cross-sectional study at a various stages of pregnancy in swden.Acta Obstet Gynecol Scand. 2009; 88(7) :807-13.

7. Ryding EL.Psychosocial indications for cesarean section.A retrospective study of 43 cases.Acta Obstect Gynecol Scan. 1991; 7(1):44-9.

8. Sasito T,Nurmi JE,Halmesmaki E,et al.Psychosocial characteristics of women and their partner fearing vaginal childbirth.BJOG. 2001; 108(5):492-8

9. Ryding EL,Wijma B,Wijma K,Rydhstrom H.Fear of childbirth during pregnancy may increase the risk of emergency cesarean section.Acta Obstet Gynecol Scand. 1998; 47(5):54247

10. Rouhe H,Salmela-Aro K,Halmesmaki E,Saisto T.Fear of childbirth according to parity,gestational age, and obstetric. BJOG.2009; 116(1):67-73.

11. Hildingssonn I,Radestad I,Rubertsson C.Few women wish to be delivery by cesarean section.BJOG. 2002; 109(6):618-23. 